\title{
Ventricular extrasystoles with syncopal episodes-perodactyly-Robin sequence syndrome
}

INSERM

\section{Source}

INSERM. (1999). Orphanet: an online rare disease and orphan drug data base. Ventricular extrasystoles with syncopal episodes-perodactyly-Robin sequence syndrome. ORPHA:3201

This syndrome is characterized by cardiac arrhythmias (ventricular extrasystoles manifesting as bigeminy or multifocal tachycardia with syncopal episodes), perodactyly (hypoplasia and/or agenesis of the distal phalanges of the toes) and Pierre-Robin sequence (see this term). 